
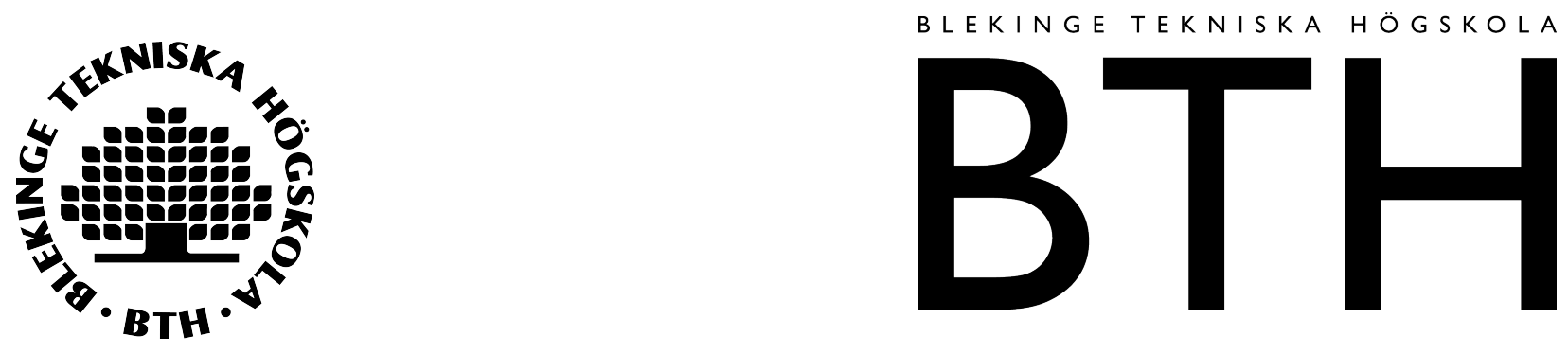

Master's Degree Thesis Computer Security | ISRN: BTH-AMT-EX--2016/CIM-01--SE

\title{
Detecting Crime Series Based on Route Estimation and Behavioral Similarity
}

\section{Johan Eliasson}

Blekinge Institute of Technology, Karlskrona, Sweden 2016 




\section{Abstract}

Context. A majority of crimes are committed by a minority of offenders. Previous research has provided some support for the theory that serial offenders leave behavioral traces on the crime scene which could be used to link crimes to serial offenders.

Objectives. Our objective was to investigate to what extent we could use route estimations and behavioral data to detect serial offenders.

Method. To investigate if we could find crime routes with high similarity we ran experiments using behavioral data from authentic burglary reports. We also used used burglary reports from serial offenders to investigate to what extent we could detect serial offender crime routes.

Results. The result show that crime series with the same offender on average had a higher behavioral similarity than random crime series. We also found crime series with high similarity without a known offender which could be interesting for law enforcement to investigate further.

Conclusion. Our results suggest that it is possible to detect crime series committed by the same offender using behavioral data from the burglary. There is also potential for the law enforcement to detect interesting crime series with high similarity using behavioral similarity.

Keywords: Linking crimes, burglary, behavioral similarity, crime series 



\section{Nomenclature}

\section{Acronyms}

\begin{tabular}{llr}
\hline DSS & Decision Support System & 1 \\
FN & False Negative & 15 \\
FP & False Positive & 15 \\
GIS & Graphical Information System & 6 \\
MO & Modus Operandi & $\mathrm{v}, 2$ \\
TN & True Negative & 15 \\
TP & True Positive & 15 \\
\hline
\end{tabular}




\section{List of Figures}

4.1 View for creating a task. . . . . . . . . . . . . . . . 9

4.2 All burgles found within the timespan and along the route are represented as a red marker. All red markers together creates a burglary set. . . . . . . . . . . 10

4.3 View all burglaries in the burgle set on a relative timespan view. . . . . . . . . 10

4.4 View top 10 most similar subset of burglaries. . . . . . . . . . . . . . . 11

4.5 Inspect a subset of burglaries in detail. The image shows when the user selected the most similar subset of burglaries with length $2 . \ldots \ldots \ldots \ldots$

6.1 Results for experiment $1 \ldots \ldots \ldots \ldots \ldots \ldots$

6.2 Results for experiment $2 \ldots \ldots \ldots \ldots$

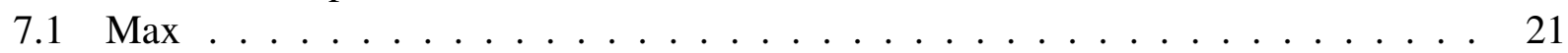

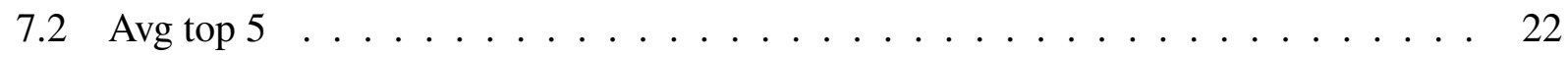

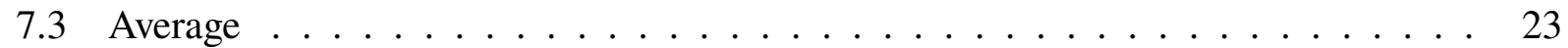




\section{List of Tables}

5.1 Categories of Modus Operandi (MO) features . . . . . . . . . . . . . . 13

5.2 Interesting route alternatives being recommended from the Swedish police. . . . . 14

6.1 Results for experiment $1 . \ldots \ldots \ldots \ldots$. . . . . . . . . . . . . . . . . . . . . . . . . . .

6.2 Results for experiment 2. . . . . . . . . . . . . . . . . . . . . 19

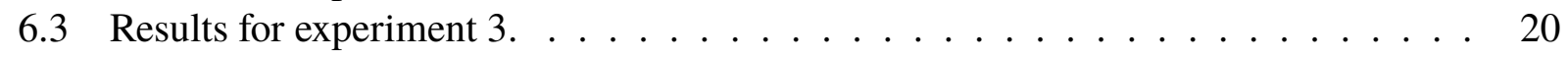





\section{Table of Contents}

Abstract

Nomenclature

Acronyms ............................ iii

List of Figures iv

List of Tables $\quad v$

Table of Contents vii

1 Introduction 1

1.1 Background . . . . . . . . . . . . . . . . . . . 1

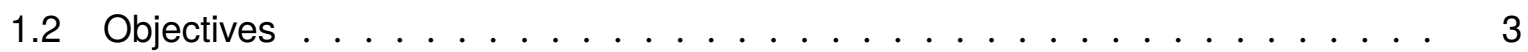

1.3 Delimitations . . . . . . . . . . . . . . . . . . . 4

1.4 Research Questions . . . . . . . . . . . . . . . . . . . . . . . 4

2 Related Work $\quad 5$

3 Algorithm $\quad 7$

3.1 Algorithm specifics . . . . . . . . . . . . . . . 8

4 Decision Support System 9

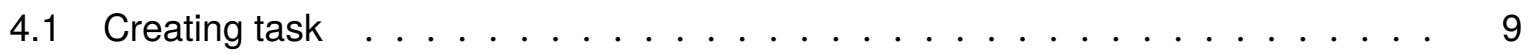

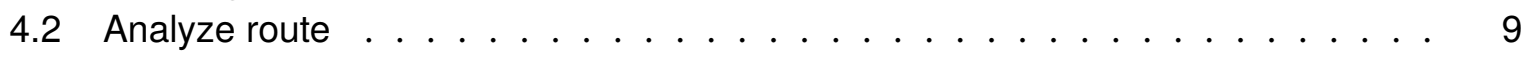

5 Method 13

5.1 Dataset . . . . . . . . . . . . . . . . . . . . 13

5.2 Routes and time span . . . . . . . . . . . . . . . . . . . 14

5.3 Measurements . . . . . . . . . . . . . . . . . . . 15

5.4 Experiment $1 \ldots \ldots \ldots \ldots \ldots \ldots$

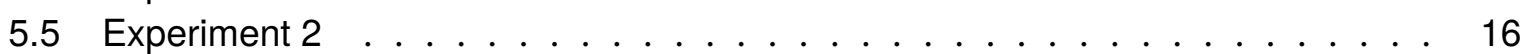

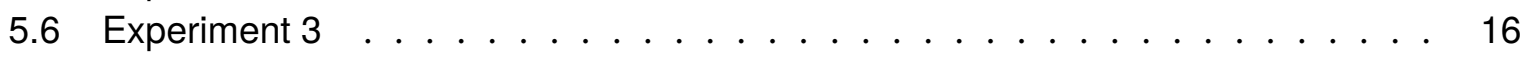

6 Results 17

6.1 Experiment $1 \ldots \ldots \ldots \ldots \ldots \ldots$

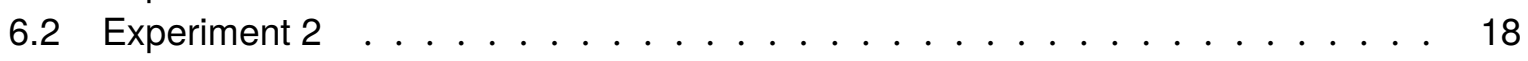

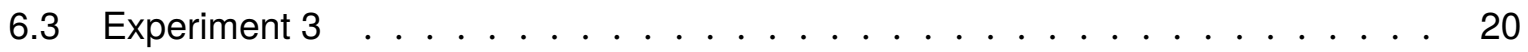

7 Discussion 21

7.1 Experiment 1 and Experiment $2 \ldots \ldots \ldots \ldots$. . . . . . . . . . 21

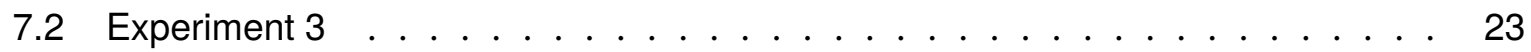

8 Conclusions 25

9 Future Work 27

References $\quad 29$ 



\section{INTRODUCTION}

In Sweden, only 3-5\% of all residential burglaries were solved during the period 2006-2016[1], indicating potential for improvement within the Swedish law enforcement on how to handle burglaries. One way of trying to solve burglaries is to target serial offenders. Serial offenders are overrepresented among all types of crimes [17].

In order for the law enforcement to efficiently target the serial offenders, the law enforcement needs to link burglaries to offenders. Linking two or more burglaries can be achieved using different methods. One way is to make use of forensic evidence such as DNA, fingerprints, tire footprints or footprints. Unfortunately, forensic evidence is present in less than $1 \%$ of crimes [17]. A complement to forensic evidence is to use behavioral features in order to identify serial offenders. The reason why behavioral features can be used is because offenders often use the same behavior when committing crimes $[17,18,10]$.

The traditional way of linking crimes would have been to have investigators do manual analysis on multiple crime reports, trying to find patterns in order to link crimes. Manual analysis is time consuming, cost ineffective and does not scale. Instead much of the linking analyzing can be automated with an algorithm [15]. An algorithm could do the analyzing and assist the investigator in making a decision on where to focus the law enforcement's resources. Such an algorithm can be part of a Decision Support System (DSS). Generally, a DSS helps and ensures a more precise decision to be made by the user of the system [15]. In the context of the thesis, a DSS would do a first analysis of reported burglaries to help the Swedish law enforcement to focus its resources on burglaries with a high behavioral similarity, i.e. potential linked burglaries, in order to improve the overall efficiency of burglary investigations.

This thesis will present and evaluate an algorithm that will identify and prioritize burgle series (crime series) based on behavioral similarity along custom routes and time spans. This algorithm will be part of a proof-of-concept web-based application. In the application the user will be able to select waypoints and a time span. The algorithm will try to find crime reports along the route that could have occured within the time span and display a list with the ten most similar crimes routes based on behavioral data. An application like this might be interesting for the Swedish law enforcement in the future, in order to analyze and prioritize crimes.

\subsection{Background}

According to the Swedish National Council for Crime Prevention[1], the clearance rate was between 3-5\% for the 23,100 reported residential burglaries in Sweden during 2014. This means that between $95-97 \%$ of all residential burglaries are never solved by the law enforcement in Sweden. A majority of these 23,100 reported residential burglaries are, as in other crime areas, committed by a minority of offenders [17], so called serial offenders.

A serial offender, who is involved in two or more burglaries, will create a so called series of burglaries. The process of identifying or linking burglaries to a series can be done using forensic evidence, also called "hard" evidence. The linking could also be done using crime scene information, also called "soft" evidence [12]. If there exist similarities in the evidence, like the same DNA is found on multiple crime scenes, those multiple crimes can be linked. However, hard evidence, such as DNA, are rarely found at crime scenes. A report from the House of Common from the United Kingdom suggests that DNA is found in less than 1\% of all crime scenes [13]. In contrast, soft evidence is always available. Soft evidence can be information such as when the crime could have been committed, i.e. temporal data. In the context of burglaries, other soft evidence data will be available. Data of where the burglary was committed, so called 
spatial data, will be available. Also different behavioral data will be available. Behavioral data for burglaries may be e.g. type of house, if the house had an alarm, method of entering, etc.

Targeting serial offenders is essential for law enforcements and for house owners. Arresting serial offenders will stop them from committing new burglaries. The clearance rate can also improve if serial offenders can be linked to multiple unsolved burglaries.

In crime theory, serial offenders often use the same technique and behavior when committing crimes $[17,10,18]$. This behavior is called Modus Operandi (MO). Behavioral data, and other soft evidence, can there for be used as an alternative to link crimes. To be able to link crimes the soft evidences needs to be both collected and organized. After that, the evidences can be analyzed to identify crimes with similarities that potentially could have been committed by the same offender.

The analysis could be handled either manually by investigators or automatically by an application. Letting human investigators manually find patterns trying to link crimes introduces difficulties in an investigation. A human cognitive capacity is limited. Cognitive shortcuts or "heuristics" are used by the human brain when trying to make a decision in a complex reality. These shortcuts may introduce biases [14]. An investigator with biases focuses on the information that confirms the biases instead of seeking evidences unconditionally. A human also has limitations in terms of work resources. An employee in Sweden typically works 40 hours a week. This however does not mean an investigator can exclusively work 40 hours per week trying to link crimes. Normally a person's workday contains overhead, including task such as reading and writing emails, attend meetings, doing planning, etc.

An alternative approach is to let an application analyze the crimes, trying to find patterns and link crimes. An application can restlessly work around the clock, processing large amount of data in a unified fashion. This enables an application to do the automated work of analyzing crime, but letting investigators having the final say and actually making the decision of what to do with the analyzed data. Such a system is called DSS.

\subsubsection{Decision Support System}

DSS started to emerge around the 1970s to assist users to make a more precise decision [15]. A DSS generally consists of three major parts.

1. Database - Managing the data from internal and external sources.

2. Model - Handles computation and analysis of the data.

3. GUI - Handles the interaction between the system and the users. The GUI enables the user to make queries, visual representations and other reports which can provide the basis for a decision.

DSS is being used in a variety of areas within business, government agencies and the military. The idea with a DSS is to analyze and organize data to assist users making a more precise and efficient decision. Problems to make a decision on can be either structured or unstructured [16]. Structured problems refer to problems that are well structured, repetitive and easily solved. Unstructured problems refer to problems that are new, ill-structured and difficult to solve. A DSS is applicable on problems containing sub problems that are both structured and unstructured. The DSS would then handle the structured part of the problem. It is then up to the human to handle the unstructured part and also to make a final decision for the whole problem.

\subsubsection{SAMS}

Today, there is an ongoing research collaborations between Blekinge Institute of Technology and the Swedish police regarding an implemented DSS, called SAMS, focusing on residential 
burglaries [6]. SAMS currently support, among other things, the collecting of spatial, temporal and behavioral characteristic data for residential burglaries. The system also has basic features such as searching and comparing burglary reports.

By facilitating searching and comparing burglary reports, SAMS assists its users to decide on what burgles the law enforcement's resources could be focused on. It is important to note that SAMS does not take any decisions on its own. It is always a human who makes the final decision.

For searches, analysis and comparison SAMS relies on data. The data within SAMS is collected and inserted by police officers across Sweden. The officers are using a standardized form for every burglary incident. The form was designed in collaboration between Blekinge Institute of Technology and the Swedish law enforcement [6]. By having the form standardized, it makes the collecting process more unified across officers and across police districts in Sweden. This makes the data comparable when it will later be used for analyzing. The data collected is soft evidence for the reported burglary which includes temporal, spatial and MO data. The data for a crime report can be collected by a police officer out on the field and later inserted into SAMS making the crime report fully accessible within SAMS and all SAMS features.

\subsubsection{Limitations}

The limitation of burglary reports with known offender, i.e. labeled data, is limited in two ways. First, not all reported crimes are solved, and second, not all solved crimes gets updated with a known offender in SAMS. Since we got data from SAMS for the experiments, we have limited access to labeled data.

Another limitation concerns burglary reports. Not all districts in Sweden uses SAMS which limits the burglaries to by located only in the districts where SAMS are used. Also, in districts where SAMS are used, it is optional for law enforcement officers to use it. This means that there is a gap between the actual reported burglaries to the Swedish law enforcement and the burglary reports within SAMS.

\subsection{Objectives}

This thesis has two major objectives.

1. Investigate to what extent we can detect serial offenders using route estimations and behavioral similarity.

2. Develop a proof-of-concept web-based application for crime route analysis.

Investigating to what extent we can detect serial offenders are interesting because the clearance rate for burglaries are below 5\% yearly, in Sweden. The law enforcement needs to analyze more crime reports trying to link burglaries to serial offender, if the law enforcement want to improve the clearance rate. We will investigate if a type of DSS can assist the law enforcement in that process.

The work of this thesis will also result in a proof-of-concept web-based application. The web-based application will enable users to list burglaries along a given route. The user will be able to specify any given route with up to 8 waypoints. The user must also specify a given time frame for when the burgles could have occurred. With both a route and a time span, the application will search through the database of burglaries. The burglaries found along the route and in the given time span will be visualized on a map in the web interface. The burglaries will also be grouped in every possible combination, creating different crime routes. Those crime routes will then be sorted on similarity and displayed for the user. The user can then choose to display specific crime routes with the corresponding burglaries data. It is possible that, in the 
future, this proof-of-concept application could be integrated into SAMS for the Swedish law enforcement to use in their day-to-day work.

\subsection{Delimitations}

For experiment 1 and experiment 2 we introduced a threshold in our algorithm. The threshold is, if a task finds more than 20 crimes, that task will be ignored. This is because the algorithm takes significantly more time to process a task with more than 20 crimes. Excluding tasks with more than 20 crimes will make the experiment's execution time reasonable for the scope of this thesis.

Another delimitation is the routes and time spans suggested by the Swedish law enforcement. An unlimited amount of routes and time spans could have been added, but to keep the experiment execution times down only 13 routes and 52 time spans was chosen. The routes and time spans are described in section 5.2. However, the routes and time spans are suggested because they are realistic and covers normal police investigator's use cases. The routes and time spans option only concerns experiment 1.

\subsection{Research Questions}

RQ I Given unlabeled data, to what extent is the algorithm capable of detecting possible crime routes with a similarity between crimes distinct from an average group of crime samples?

To answer RQ I, we will evaluate crimes without a known offender. This similar to how the Swedish law enforcement is working when trying to find similar burglaries. In order to answer this question, we run an experiment where we will be identifying multiple crime routes and look how crime routes can vary in similarity from an average crime route.

RQ II Given labeled data, to what extent is the algorithm capable of detecting possible crime routes with a similarity between crimes distinct from an average group of crime samples?

RQ I and RQ II are similar. The two experiment will be evaluated the same way and generate the same type of result. When running the experiment to answer this research question we will use only crime reports with known offenders. Previous research suggests that crimes with the same offender can generate crime route series with higher similarity than a random sample $[17,10,18]$.

RQ III Given labeled data, what accuracy can be achieved using the algorithm to detect serial offender crime routes?

Previous studies suggest that a serial offender uses the same behavioral technique when committing burglaries. RQ III is meant for testing and replicating those previous studies. To test this our experiment will evaluate routes between burglaries from known offenders. The result will give us insight to what extent our algorithm can differentiate burglaries committed by a known offender, from burglaries committed by other offenders. 


\section{RELATED WORK}

B. and Fosdick [2] describes that, around the year 1930, the main reason to why the police are able to connect crimes to criminals is because of luck and guesses. A thought on a more systematically approach started to emerge. When interviewing the formerly Commissioner of the City of London Police, Sir Henry Smith, he described that thieves follows repetitive patterns. B. and Fosdick also suggest that every criminal leave individual traces, i.e. MO features. By identifying the traces, it should be possible to connect traces to criminals.

Woodhams et al. [18] did a literature review on the topic of linking crime using behavioral similarity. They found that the current research support, to some extent, the assumption that it is possible to use behavioral similarity to link a crime to an offender. However, the authors note that not all behaviors shows the same potential to be used for crime linking. They also say that the research supporting crime linking are limited and that there is a need for replication studies and studies looking at different types of crimes.

Bennell and Canter [3] look at what behavioral features can be used in order to objectively detect linked and unlinked crime pairs. They also brought up how the police can weight false positive linked crimes, stating that "a police force may decide it is ten times more important to make correct decisions when faced with linked crimes compared to unlinked crimes". Their results indicate that the distance between two burglaries is the most stabile aspect of feature when it comes to linking crimes. Other features were also included in the study but those other features were not as likely to link crimes as the distance between burglaries. Three years later, Bennell and Jones [4] did a similar study, trying to replicate the previous study with new data. They found similar results. Again the distance between burglaries was the best feature for linking crimes. They also added that other types of features were feasible for linking such as what things were stolen.

Bernasco [5] talks about how initially it is easy to assume that it is the same offender, if the same house is burgled twice within a short time span. This assumption is strengthened in their study, were there results indicates that it is a higher probability that it is the same offender for burglaries occurring in short distance, both in time and space. They found this results by analyzing 3624 burglaries during 1996-2004 in and around The Hague. The author also raises a warning of the differences that exist between burglaries that are reported, compared to burglaries that are never reported.

Tonkin et al. [17] looked at to what extent they could successfully classify crimes as linked or unlinked across different types of crimes. They looked at violent, sexual, and property-related offenses. Their results indicated that both intercrime distance and temporal proximity were good indicator for if crimes were linked or unlinked.

Markson et al. [10] studied 160 crimes to see if they too could find evidence that different types of soft evidence could be used to link crimes. They found that only the spatial and temporal data was feasible to link crimes. They used three behavioral features (entry behavior, property stolen and target selection) and their conclusion was that none of those features, individually or combined, could be used to link crimes.

Iwanski et al. [8] looked at the relationship of offender's location of crime and the travel routes an offender takes in their average day. The author had access to the offender's home, work location and location of the crimes they committed. Given this information simulated possible travel routes of offenders were created to see if the offender's crimes could be predicted. Other than home and work, major attractors, such as shopping centers, were used as locations were the offender were likely to travel in between. The authors found that the majority of crimes were located along the simulated paths taken by the offender, indicating that offenders commit crimes 
in their everyday surrounding.

Borg et al. [6] built a DSS for the Swedish police to systematically collect, compare and analyze data from burglaries. With the help of the collected burglary data, they then applied a cut-cluster algorithm to groups crimes based on modus operandi, residential characteristics, stolen gods, spatial similarity or temporal similarity. They found that clustering algorithms performs significantly better than a random guess, indicating clustering algorithms are capable of differentiate interesting crimes for police investigators.

Oatley et al. [11] makes a very comprehensive description of the challenges of detecting, linking and preventing burglary. They describe the lack of standardized data collection and how much of the existing data only can be found in free-text, making it hard to apply data mining techniques on the data. They also talk about how different type of visual representations of data can aid investigator in decision making. The visual representation can be charts (e.g. pie or bar charts) or information on maps such as Graphical Information System (GIS). Visual representation is important because of the volume of data an investigator should manage when finding inter-crime patterns. They conclude by saying how important it is for DSS to be grounded in forensic psychology and criminology.

In summary, the psychological theories suggest that a serial offender will, to some extent, use the same behavior and technique when committing crimes. It is also suggested that a serial offender commits crimes in close proximity in both time and space. These theories could be used to link crimes. One approach would be to collect spatial, temporal and behavioral data of crimes, to analyze trying to connect crimes.

Previous studies have shown that spatial and temporal features are promising to use for linking crimes. Using behavioral features to link crimes has had some mixed results. Some studies have been able to use behavioral features to link crimes, where as other studies have been unable to draw the same conclusion.

This paper will try to link burglaries along a given route. Previous studies has shown that criminals tend to commit crimes along roads they are familiar with. In our study, we will use route and time span as search parameters to find burglary sets. This has, to our knowledge, never been done before. The burglary sets will then be analyzed for similarities in behavioral data trying to detect serial offenders. 
Our developed algorithm tries to detect and analyze crimes. The algorithm can be divided into two steps. The first step is for the algorithm to discover all routes along a given route. The second step is to prioritize the crimes based on similarity. The result of the algorithm is a list of all possible crimes along a route and prioritized lists of crimes based on similarity. See Algorithm 1 for a detailed description of the algorithm.

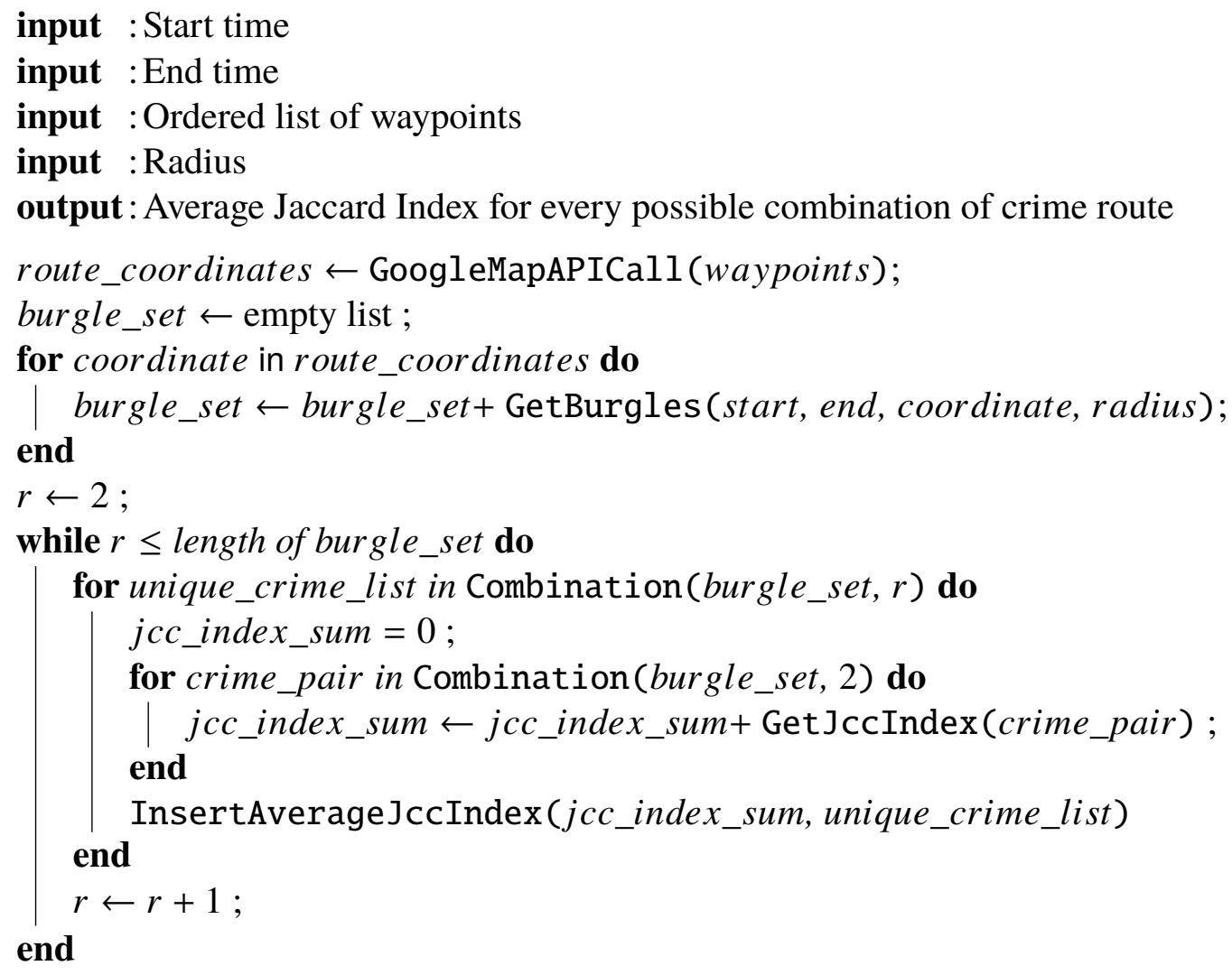

Algorithm 1: Calculate Jaccard Index for all possible combination of crime routes within a burgle set.

The algorithm takes in a start time, end time, ordered list of waypoints and a radius as arguments. Given the list of waypoints, the algorithm gets the quickest route between the waypoints using Google Maps API. Google Map API returns the quickest route in the form of a list of coordinates which is placed consecutively along the route. The algorithm then steps through all coordinates along the route and for each coordinate search the database for crimes that is both within the timespan and that is within the radius of the coordinate on the route. All discovered burglaries are placed in a set, later referred to as a burglary set, for analysis.

The analysis of burglaries in the burglary set involves prioritize, based on similarity, all combinations of routes within the burglary set. The similarity is calculated using Jaccard Index [9]. Using Jaccard Index is in line with previous studies[10] that has also used Jaccard Index to measure behavioral similarity in binary data. The Jaccard Index is calculated as followed:

$$
\operatorname{JCC}(A, B)=\frac{|A \cap B|}{|A \cup B|}
$$

$A$ and $B$ represent finite sets of boolean MO features for individual crimes. For every combination of crime route a similarity is being calculated. The similarity is being calculated 
by taking the average Jaccard Index of all combinations of two pair crimes in the current crime route.

\subsection{Algorithm specifics}

All MO features are stored using one of three values; True, False or Null. True is being used when the MO feature is present. False is being used when the MO feature is not being present and Null is being used when there is an uncertainty if the MO feature should be True or False. Since the Jaccard index only works with boolean variables (True and False) the MO features across burglaries in a burgle set needed to be handled. To handle MO features, only MO features that are present as True or False in all burglaries within a burgle set are being used to calculate the Jaccard index.

Google Map API in used to find a route between two or more waypoints. Other methods could have been used, such as implement our own route service algorithm. However, to implement our own service to receive routs was out of the scope of this thesis. The reason to why we chose Google Maps API was to quickly get a route given two or more waypoints. Google Maps API is also free up to 2,500 requests per day with the ability to upgrade to a paid plan with capacity of 100,000 request per day.

The Combination function in the algorithm takes two arguments. A list $l$ and an integer $n$. The function then returns all subsets (order does not matter) with length $n$ of the list $l$. For a burgle set, the total number of crime routes involving two or more burglaries is described in Equation 3.2 where $N$ is the number of burglaries found along the route.

$$
\sum_{k=2}^{N} \frac{N !}{k !(N-k) !}
$$

InsertAverage JccIndex is a function in Algorithm 1 that inserts all possible burglary series and the burglary series Jaccard index, i.e. how similar, based on behavioral data, the series is. 


\section{DECISION SUPPORT SYSTEM}

Below follows a description of the proof-of-concept web based application that was developed during this thesis. The application is meant to be used by police investigators, assisting investigators differentiate interesting burglaries with high similarity. The application is based on SAMS database of burglary reports.

\subsection{Creating task}

In the application, a user can create a task. To create a task, the user specifies a timespan and a route. The timespan is between two arbitrary dates. The route has a origin, a destination and up to 6 waypoints. This enables the user to narrow the search in time and space. The route is immediately displayed in on the map so the user can make changes to the route if necessary. In the example in figure 4.1 the timespan is between 2014-01-01 - 2014-04-30 and the route is between Karlskrona and Karlshamn with two waypoints; Ronneby and Stengrepen.

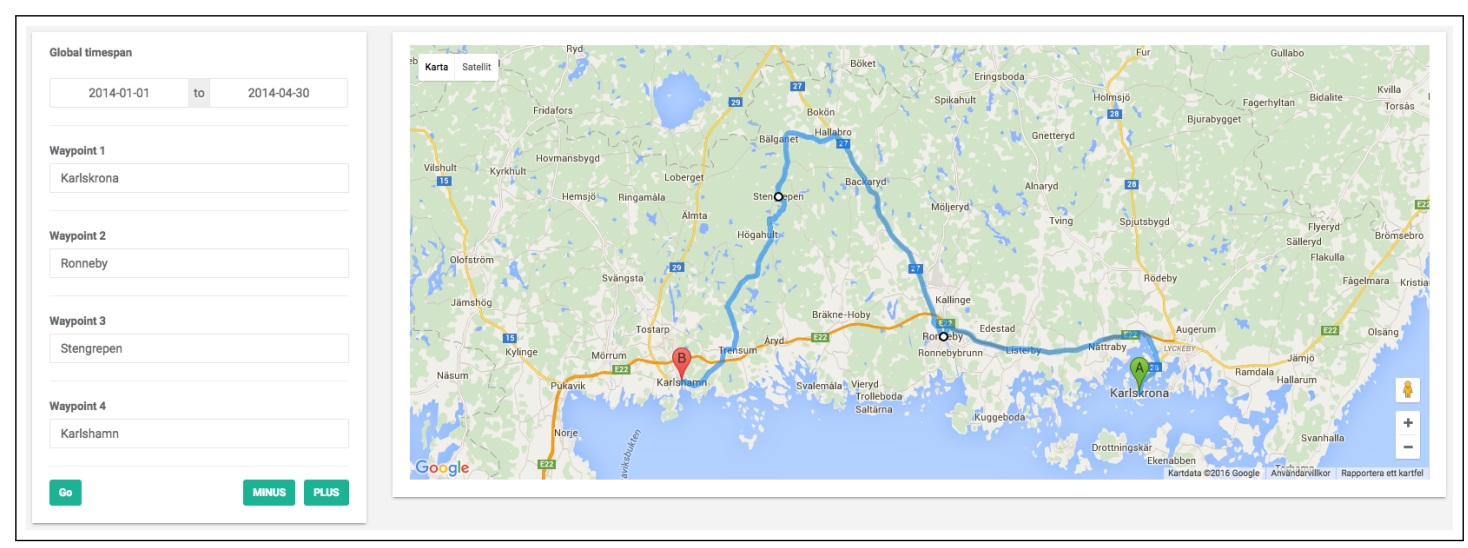

Figure 4.1: View for creating a task.

\subsection{Analyze route}

Once the task is created Algorithm 1 starts processing the task. When the algorithm is done computing, the user can further analyze the result within the application. The route is present on a map with red markers along the route. The green marker with the letter "A" represents the starting point of the route. The red marker with the letter " $B$ " represents the ending point of the route. All other red markers represent a burglary. 


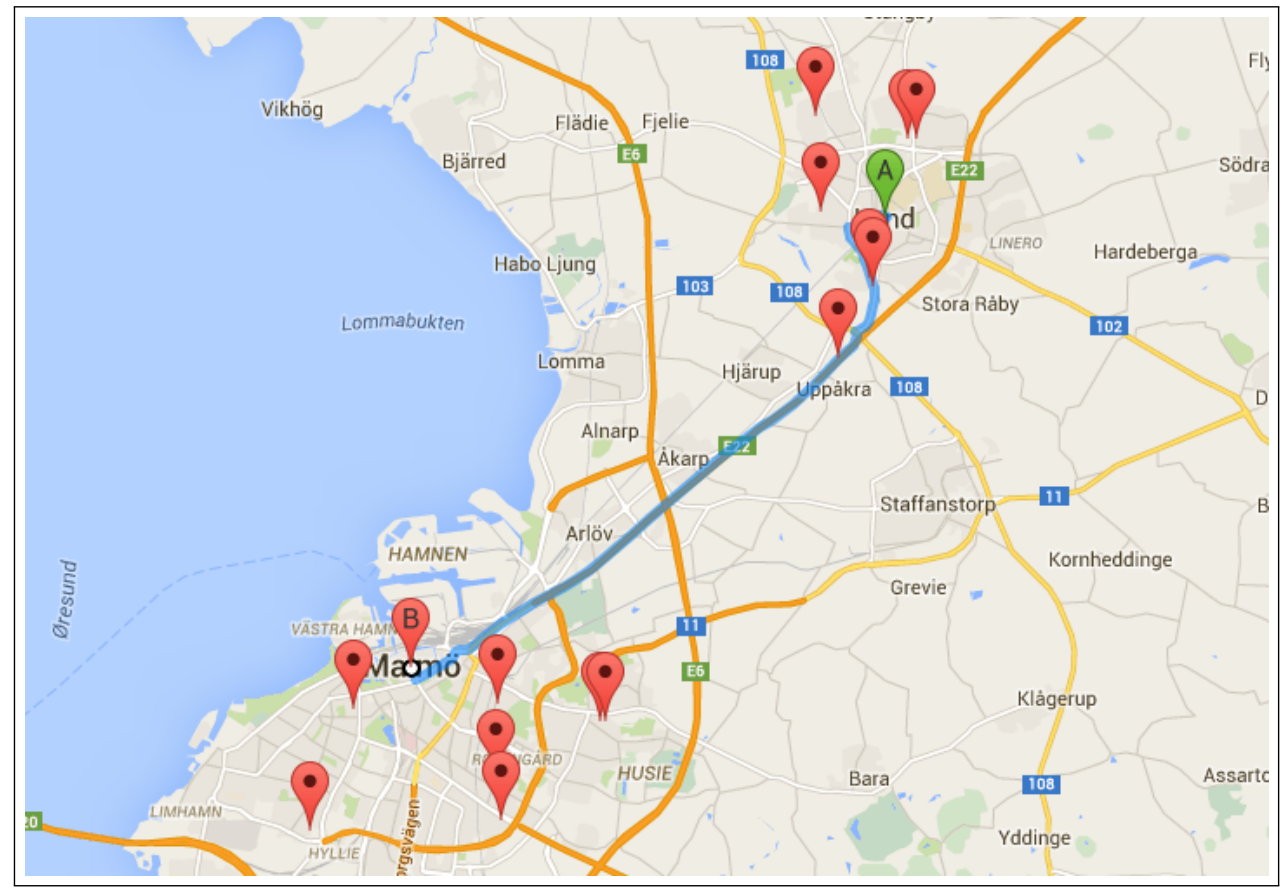

Figure 4.2: All burgles found within the timespan and along the route are represented as a red marker. All red markers together creates a burglary set.

Below the map are two tabs. Timespan and Similarity. The timespan tab shows green rectangles for each burglary and represent, relative to the total timespan search, when a burglary could have been committed. When a user hover a green rectangle with the cursor the corresponding red marker for the burglary starts bouncing. This way, a user can hover the green rectangles and get a perception of how the burglaries on the map are related to each other in time.

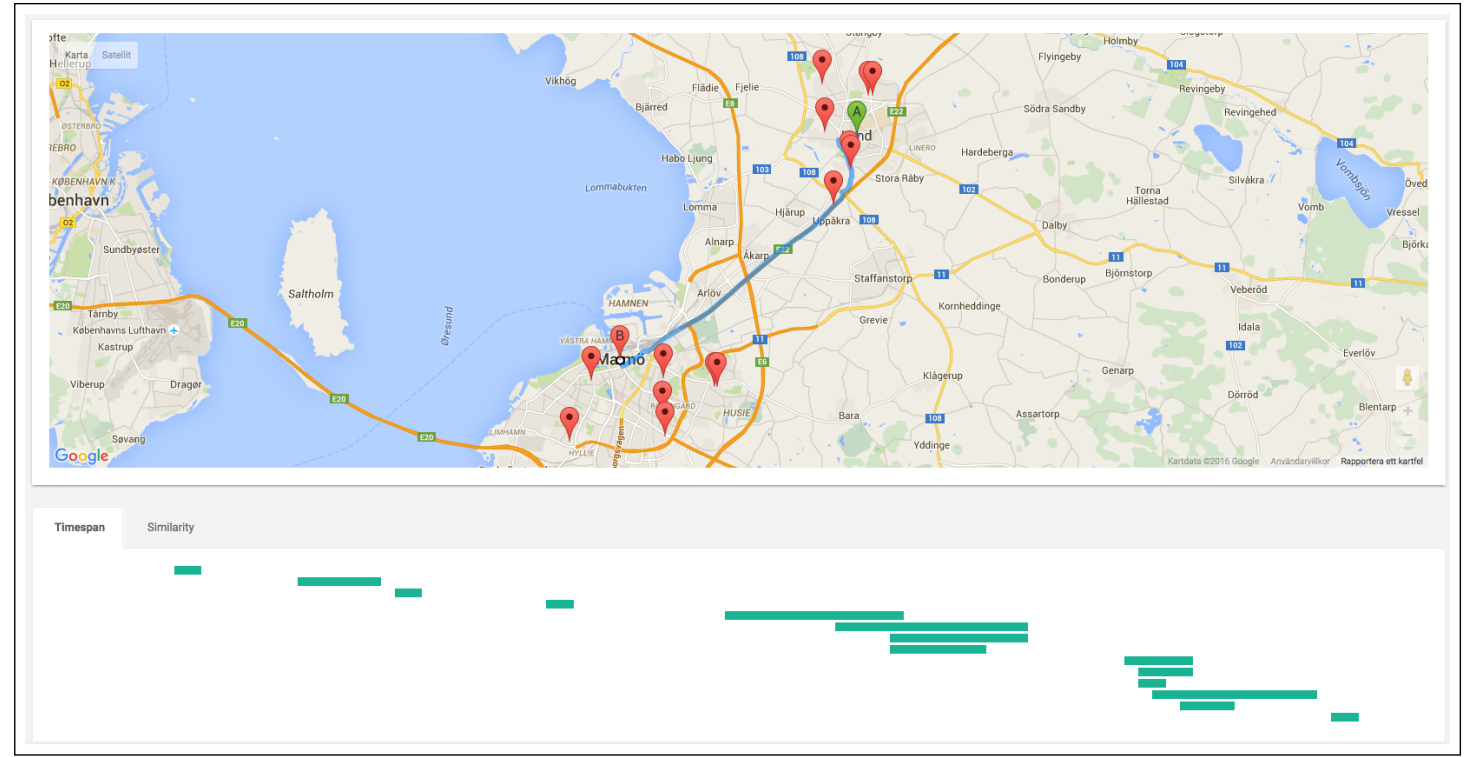

Figure 4.3: View all burglaries in the burgle set on a relative timespan view.

The similarity tab displays the top 10 most similar burglaries for each subset. The subsets starts with length 2 and ends with length $n$ where $n$ is the length of the burglary set. This view enables users to get a quick overview of the overall similarity for the most similar burglary 
subsets. In this stage, the algorithm has provided the user with interesting burglary subsets to analyze further.

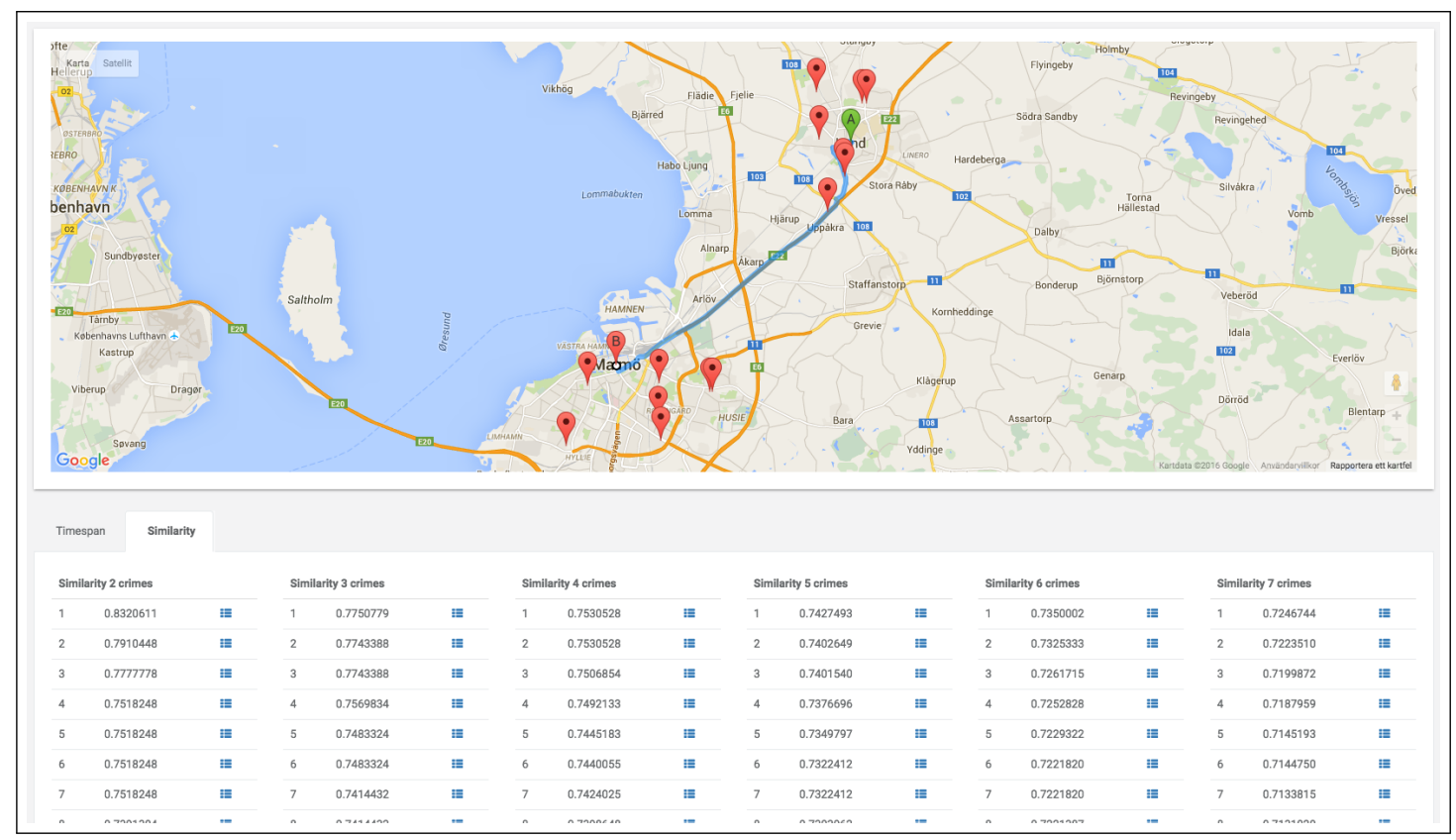

Figure 4.4: View top 10 most similar subset of burglaries.

If the user decides to further analyze a subset of burglaries, the user can click on the row of a burglary subset. In Figure 4.5 the user has clicked the most similar burglary subset containing 2 burglaries with a similarity of 0.832 . When the user click on the burglary subset, the map will zoom in on the burglaries in the subset and mark all burglaries in the subset with a green marker. The user can then get information of all burglaries in the subset for further analysis.

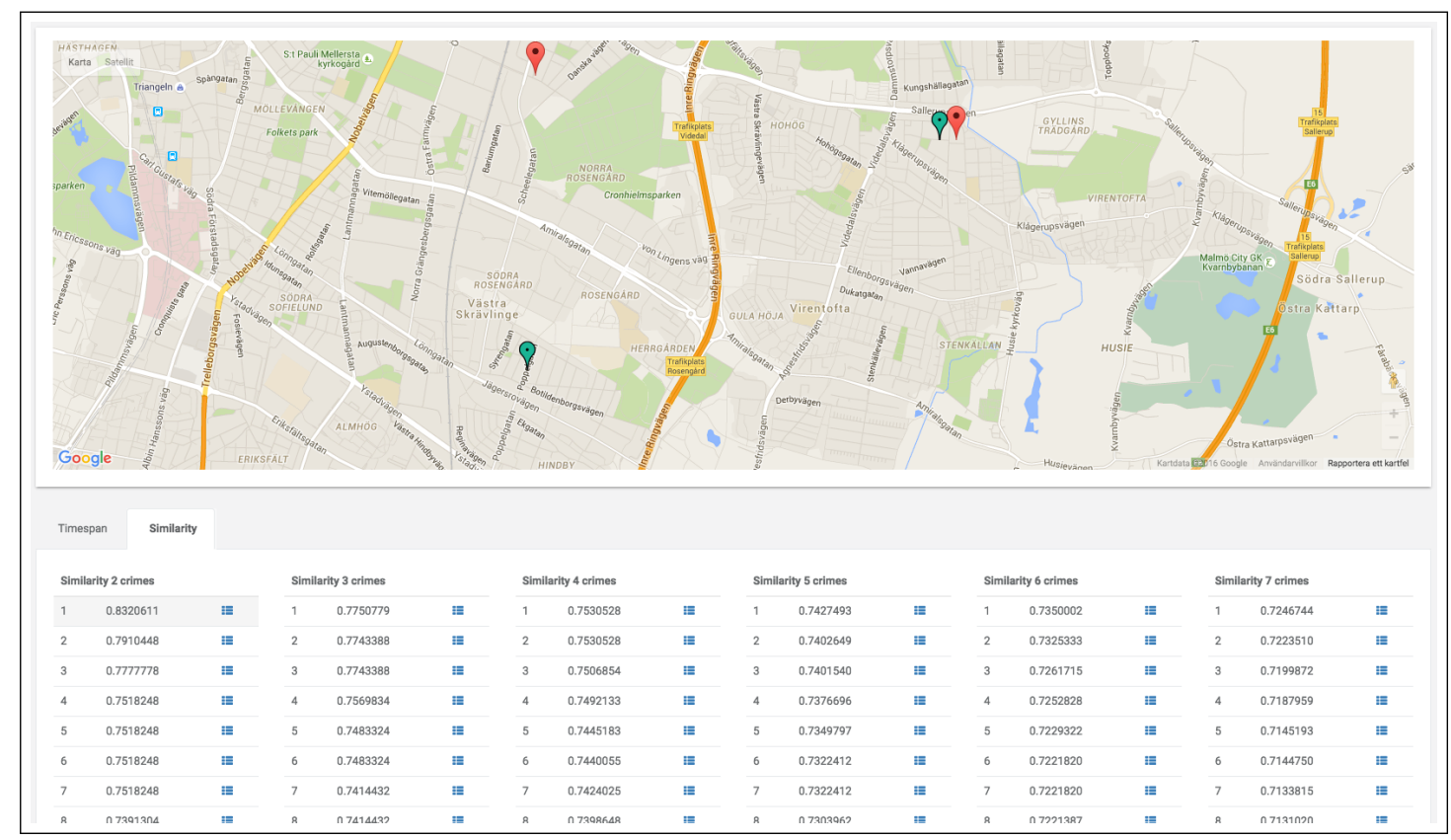

Figure 4.5: Inspect a subset of burglaries in detail. The image shows when the user selected the most similar subset of burglaries with length 2 . 
To summaries, the application allows users to search for burglaries in time and space. The algorithm then provides the user with subsets of burglaries with a high similarity for the user to further analyze. This is interesting for police investigators because it can help them find interesting crimes without much effort. The application handles the processing of burglary reports and the investigators can make a final decision on what burglaries to focus police resources on. 
A total of three experiments will be run. The first two experiments try to detect routes with a similarity between crimes distinct from an average group of samples. The first experiment uses unlabeled data whereas the second experiments uses labeled data. The third and last experiments will measure the accuracy achieved by our algorithm to detect serial offender routes. All three experiments will use the algorithm described in section 3. Also, all three experiment will skip tasks with more than 20 burglaries as described in the Section 1.3.

The algorithm take a total of four arguments. The first two arguments are start time and end time. The third argument is a waypoint (route) and the last argument is radius search size. The first three arguments are experiment specific. The last argument, radius, is fixed for all experiments. We will use $2 \mathrm{~km}$ as the radius of the search area, meaning only crimes closer than $2 \mathrm{~km}$ from a route will be included in the algorithm. Previous studies[8] has suggested that most crimes can be found in less than $1 \mathrm{~km}$ from commonly used roads. However, that study was concerning with city areas. Our burglary data is both in cities and on the countryside, hence the larger radius search space.

\subsection{Dataset}

The dataset used in all three experiments originate from the database of burglary reports in SAMS. In SAMS, there exist one database post per inserted burglary report. The inserted burglary reports contains MO features for the current burglary. All MO features has one of three values: True, False or Unknown. The algorithm and how the algorithm handles MO features are described in section 3 .

There are $9 \mathrm{MO}$ categories. A number of MO features are present under each category. The specific MO categories are listed in Table 5.1.

Table 5.1: Categories of MO features

\begin{tabular}{lr}
\hline Category & Features \\
\hline \hline Type of residential area & 5 \\
Alarm & 4 \\
Object description & 8 \\
Plaintiff & 16 \\
Access objects & 16 \\
Identified search & 3 \\
Goods & 14 \\
Trace evidence & 8 \\
Other & 4 \\
\hline
\end{tabular}

To summarize, a total of $78 \mathrm{MO}$ features exists.

\subsubsection{Unlabeled data}

Unlabeled data is used for experiment 1 and consists of all crimes without concerning about who the offender to those crime was, i.e. all burglary reports without any connection to who the offender was. 


\subsubsection{Labeled data}

Experiment 2 and experiment 3 will use labeled data, meaning crime series where the offender is known. In our dataset we have 9 crime series where the offender is known. When using labeled data, each crime series will be a separate task. The task's start location and star time will be set to the location and star time for the chronologically first burglary committed by the current offender. The task's end location and end time will be set to the location and end time of the chronologically last burglary committed by the offender. This will create a task for the algorithm to work on, with a given timespan and a given start and end location for a route.

\subsection{Routes and time span}

To get realistic cases for routes and time spans when running experiment 1 with unlabeled data we have received recommendations from the Swedish police on what routes and time spans to run the experiment with.

\subsubsection{Routes}

The reason behind the chosen routes was to have a variation of routes. The following routes cover routes between cities across counties, routes between cities in the same county, routes between a small village and close located city and routes between small villages. The routes was recommended by the Swedish police.

Table 5.2: Interesting route alternatives being recommended from the Swedish police.

\begin{tabular}{lr}
\hline Route & Distance $(\mathrm{km})$ \\
\hline \hline Routes between cities across counties & \\
Malmö - Göteborg & 271 \\
Karlskrona - Växjö - Jönköping & 226 \\
Karlskrona - Växjö - Halmstad & 239 \\
Karlskrona - Kristianstad - Halmstad & 265 \\
Routes between cities in the same county & \\
Lund - Malmö & 19 \\
Karlskrona - Kalmar & 89 \\
Karlskrona - Karlshamn & 56 \\
Karlskrona - Hässleholm & 141 \\
Routes between a small village and a close located city & \\
Karlskrona - Jämjö & 20 \\
Kristianstad - Åhus & 18 \\
Skurup - Ystad & 20 \\
Routes between small villages & \\
Dalby - Sjöbo & 25 \\
Älmhult - Tingsryd & 60 \\
\hline
\end{tabular}

\subsubsection{Time span}

Experiments will run on data for a whole year in time spans. A time span is one week, i.e. from Monday 00:00 to Sunday 23:59. 52 time spans will be used starting from Monday the 6th January 2014. 2014 was chosen because it was the year with most crime reports available. 


\subsection{Measurements}

\subsubsection{Similarity}

For each burglary set, the algorithm will try all possible combinations of crime routes, creating new burglary subsets. Subsets will have the length $k$ where $2 \leq k \leq n$ where $n$ is the length of the original burglary set. Two values will be calculated for all burglary subsets with length $k$ within an experiment. Avg top 5 and Max.

Avg top 5 - Average similarity, and standard deviation, of the top 5 most similar crime routes of all burglary subsets. The reason to why we chose top 5 was because of recommendations from the Swedish police.

Max - The crime route with the highest similarity of all burglary subsets.

A third value that will also be calculated for an experiment is the average similarity.

Average - Average similarity (Jaccard index) for all burglary sets.

\subsubsection{Accuracy}

Accuracy, precision and recall are used to measure experiment 3. To calculate these three values, we will introduce True Positive (TP), True Negative (TN), False Positive (FP), False Negative (FN). TP is a burglary committed by the current offender and the burglary is found in the burglary set. TN is a burglary that is not committed by the current offender and the burglary is not found in the burglary set. FP is a burglary not committed by the current offender and the burglary is found in the burglary set. FN is a burglary committed by the current offender and the burglary is not found in the burglary set.

Accuracy is the percentage of correct labeled burgles. Accuracy is calculated as:

$$
\text { accuracy }=\frac{T P+T N}{T P+T N+F P+F N}
$$

Precision is the percentage of selected burglaries that are correct. Precision is calculated as

$$
\text { precision }=\frac{T P}{T P+F P}
$$

Recall is the percentage of correct burglaries that are selected. Recall is calculated as

$$
\text { recall }=\frac{T P}{T P+F N}
$$

$F_{1}$-score is often used when TN abounds[7]. This makes the $F_{1}$-score useful when TN are not relevant. $F_{1}$-score is calculated as

$$
F_{1}=2 \cdot \frac{\text { precision } \cdot \text { recall }}{\text { precision }+ \text { recall }}
$$

\subsection{Experiment 1}

Experiment 1 will try answer RQ I were we evaluate unlabeled data in order to detect possible crime routes distinct from an average group of crime samples. To do this, we will use the dataset described in Section 5.1, the routes described in Section 5.2.1 and the time span described in Section 5.2.2. This will result in examine 13 routes over 52 weeks each, generating a total of 676 tasks. Experiment 1 will be measured using similarity as described in Section 5.3.1. 


\subsection{Experiment 2}

Experiment 2 will try answer RQ 2 were we evaluate labeled data in order to detect possible crime route distinct from an average group of crime samples. To do this, we will use the dataset described in Section 5.1 and use tasks with known offender as described in Section 5.1.2. This will give us a total of 9 tasks, since that is how many offenders that has committed 3 or more crimes, that exists in the SAMS database. The measurements mentioned in Section 5.3.1 will be used.

Experiment 2 is an extension of experiment 1. Both experiments are measured with the same methods, what differs is the dataset of burglaries. Instead of looking at specific routes and weeks as time spans as in experiment 1, experiment 2 will instead use labeled data to build its tasks on.

\subsection{Experiment 3}

Experiment 3 will try to answer RQ 3 were we evaluate labeled data to detect serial offender routes. To do this, we will use the dataset described in Section 5.1 and use tasks with known offenders as described in Section 5.1.2. The experiment will be measure accuracy described in Section 5.3.2. We will also measure the similarity of the burglary set that our algorithm picks up (TP and FP). We will then see how the $F_{1}$-score and the Similarity correlate using the Spearman's rank correlation coefficient.

The Spearman's rank correlation coefficient is calculated as

$$
r_{s}=1-\frac{6 \sum d_{i}^{2}}{n\left(n^{2}-1\right)}
$$

where $d_{i}$ is the difference between paired ranks for each observation and $n$ is the length dataset. $r_{s}$ goes from -1 to 1 . The higher $\left|r_{s}\right|$ is, the stronger the correlation is.

Experiment 3 is an extension of experiment 2, using the same dataset as experiment 2 with different measurements. Experiment 3 will instead look at how the algorithm performs when trying to find authentic crime routes with burglaries committed by the same offender. 


\section{RESULTS}

\subsection{Experiment 1}

Experiment 1's result can be seen in figure 6.1 and table 6.1. The results shows that our algorithm is capable of detecting burglaries with a higher similarity, based on MO features, distinct from a random crime route with 2-15 burglaries within a crime route. Crime routes with 16-20 burglaries are on average below the average in similarity. The crime routes with highest similarity are significantly higher than both the average and the avg top 5 .

Note that Length in the header of table 6.1, 6.2 and 6.3 is burglary subset length.

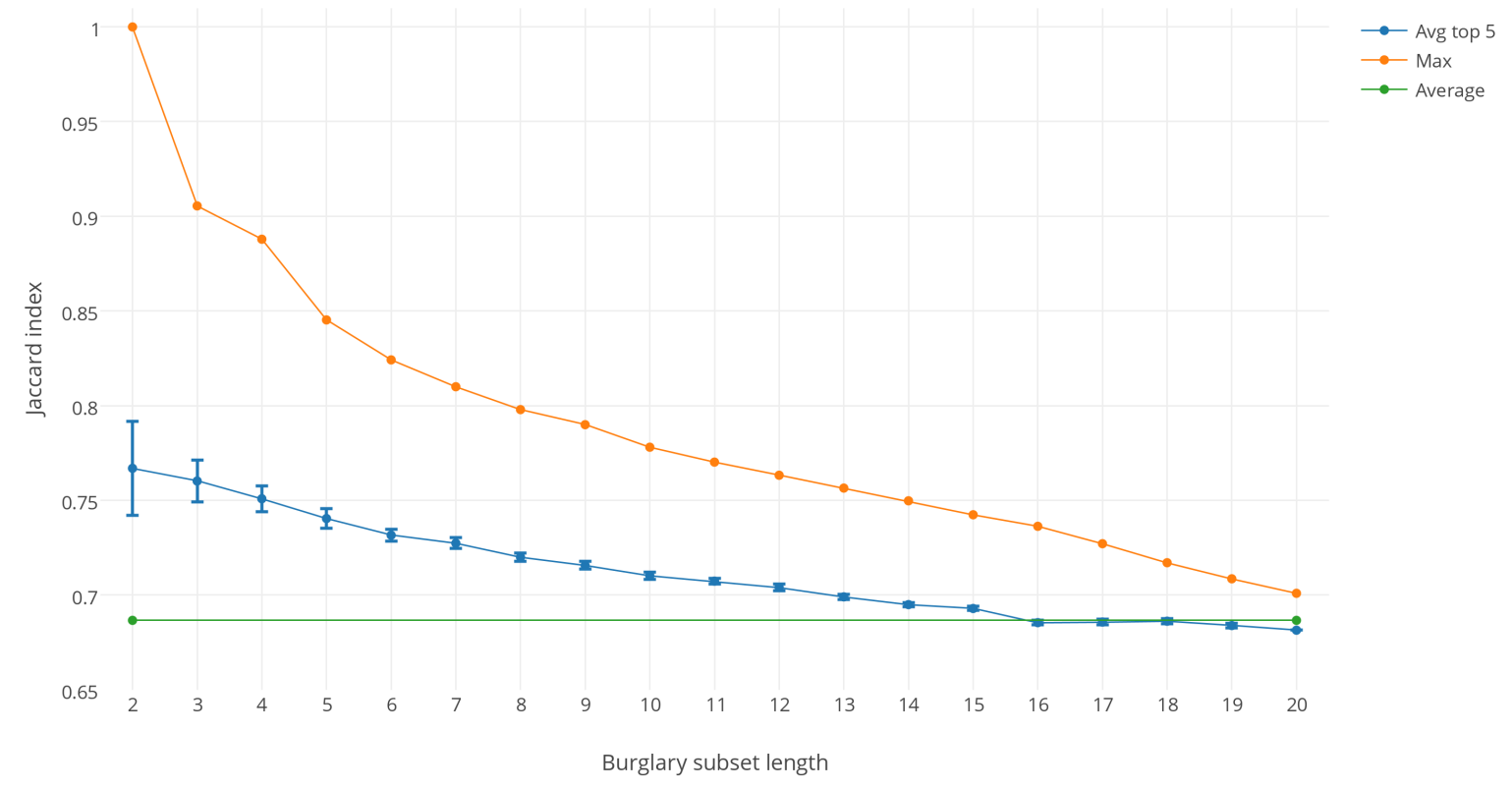

Figure 6.1: Results for experiment 1. 
Table 6.1: Results for experiment 1.

\begin{tabular}{ccc}
\hline Length & Top 5 avg similarity & Highest \\
\hline \hline 2 & $0.7669(0.0249)$ & 1.0000 \\
3 & $0.7602(0.0111)$ & 0.9055 \\
4 & $0.7508(0.0068)$ & 0.8879 \\
5 & $0.7404(0.0051)$ & 0.8453 \\
6 & $0.7316(0.0032)$ & 0.8242 \\
7 & $0.7274(0.0029)$ & 0.8100 \\
8 & $0.7200(0.0022)$ & 0.7979 \\
9 & $0.7156(0.0020)$ & 0.7899 \\
10 & $0.7102(0.0018)$ & 0.7780 \\
11 & $0.7073(0.0016)$ & 0.7702 \\
12 & $0.7040(0.0017)$ & 0.7632 \\
13 & $0.6990(0.0013)$ & 0.7565 \\
14 & $0.6949(0.0010)$ & 0.7497 \\
15 & $0.6929(0.0011)$ & 0.7424 \\
16 & $0.6854(0.0012)$ & 0.7363 \\
17 & $0.6857(0.0016)$ & 0.7271 \\
18 & $0.6862(0.0013)$ & 0.7170 \\
19 & $0.6839(0.0012)$ & 0.7085 \\
20 & $0.6814(0.0000)$ & 0.7009 \\
\hline \hline Average & $0.6866(0.0383)$ & \\
\hline & &
\end{tabular}

\subsection{Experiment 2}

Experiment 2's result can be seen in figure 6.2 and table 6.2. Experiment 2 has similar results as experiment 1 . There is less data for experiment 2 than for experiment 1 , because of the limited number of labeled data (i.e. known offenders) in SAMS. Only 9 instances of labeled data could be used for experiment 2. Both Avg top 5 and Average is generally slightly higher than experiment 1's corresponding values. The Max value is lower for experiment 2 than for experiment 1. 


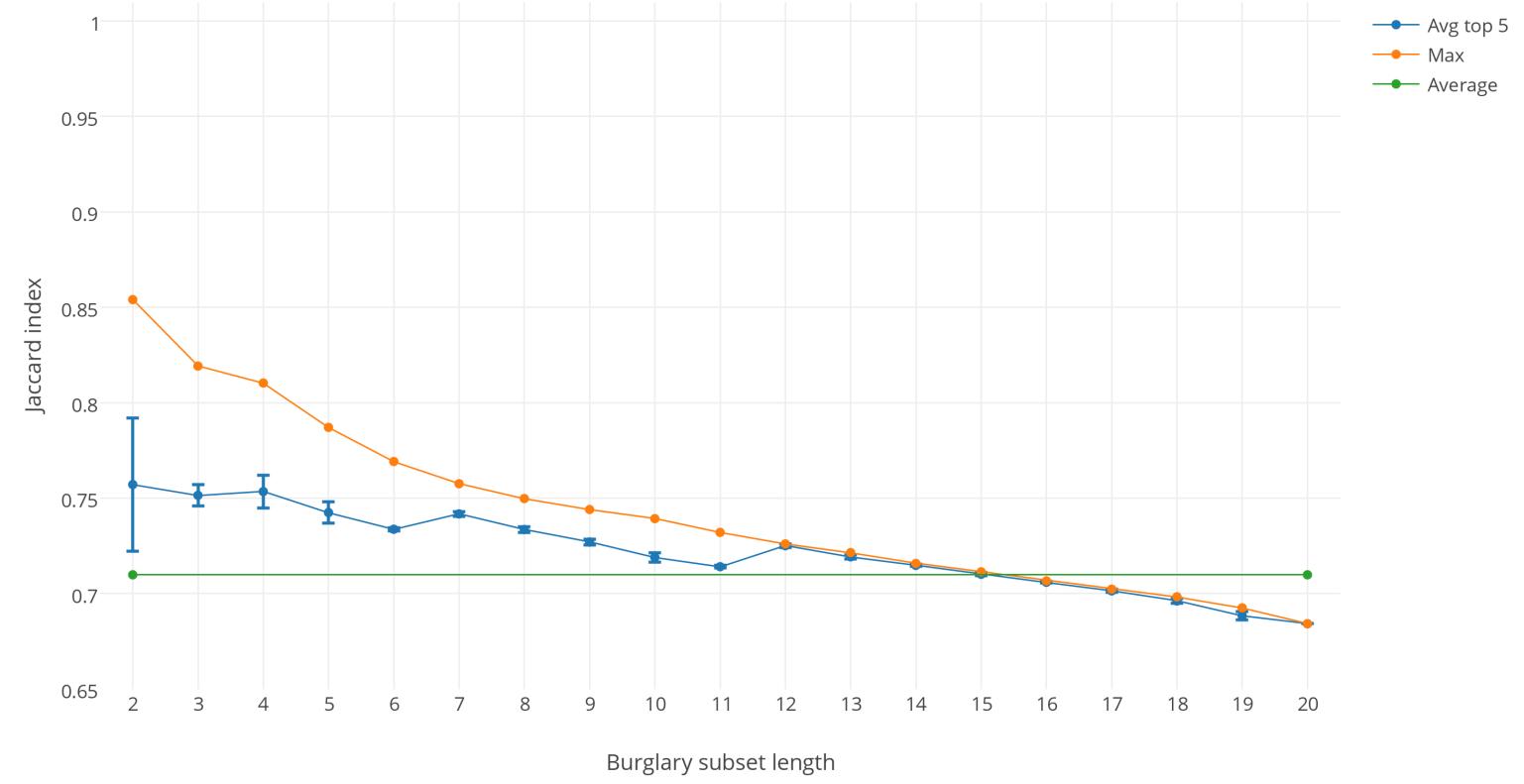

Figure 6.2: Results for experiment 2.

Table 6.2: Results for experiment 2.

\begin{tabular}{ccc}
\hline Burgles & Top 5 avg similarity & Highest \\
\hline \hline 2 & $0.7571(0.0350)$ & 0.8540 \\
3 & $0.7514(0.0055)$ & 0.8192 \\
4 & $0.7534(0.0085)$ & 0.8103 \\
5 & $0.7424(0.0055)$ & 0.7870 \\
6 & $0.7337(0.0008)$ & 0.7692 \\
7 & $0.7417(0.0013)$ & 0.7575 \\
8 & $0.7335(0.0015)$ & 0.7497 \\
9 & $0.7270(0.0015)$ & 0.7440 \\
10 & $0.7190(0.0025)$ & 0.7392 \\
11 & $0.7140(0.0007)$ & 0.7320 \\
12 & $0.7250(0.0009)$ & 0.7260 \\
13 & $0.7192(0.0011)$ & 0.7214 \\
14 & $0.7147(0.0006)$ & 0.7157 \\
15 & $0.7101(0.0008)$ & 0.7114 \\
16 & $0.7059(0.0004)$ & 0.7066 \\
17 & $0.7014(0.0008)$ & 0.7023 \\
18 & $0.6961(0.0011)$ & 0.6983 \\
19 & $0.6883(0.0022)$ & 0.6925 \\
20 & $0.6841(0.0000)$ & 0.6841 \\
\hline \hline Average & $0.7098(0.0212)$ & \\
\hline
\end{tabular}




\subsection{Experiment 3}

Experiment 3's result can be seen in table 6.3. Since we uses the same labeled data as in experiment 2 , we only have 9 offenders with 3 or more burglary reports in our data set. The low number of offenders for this experiment should be noted.

The accuracy is never below 0.99 . This is mostly because of the great number of $\mathrm{TN}$, which is an extension is a result of the method we uses for experiment 3 for how we set the start time, start location, end time and end location. This way of division the search will automatically ignore most burglaries and place most burglaries as TN. The $F_{1}$-score is more interesting to look at, since it ignores $\mathrm{TN}$.

Note that the header Similarity in table 6.3 is the similarity of the burgle set found by the algorithm (i.e. TP and FP).

A spearman's rank-order correlation was run to determine the relationship between $F_{1}$-score and Similarity. There was a strong, positive correlation between $F_{1}$-score and Similarity, which was statistically significant $\left(r_{s}=0.790, p=0.011\right)$.

Table 6.3: Results for experiment 3.

\begin{tabular}{cccccccccc}
\hline Length & TP & TN & FP & FN & Accuracy & Precision & Recall & $F_{1}$-score & Similarity \\
\hline \hline 13 & 5 & 14966 & 15 & 8 & 0.9985 & 0.2500 & 0.3846 & 0.3030 & 0.6841 \\
6 & 3 & 14988 & 0 & 3 & 0.9998 & 1.0000 & 0.5000 & 0.6667 & 0.7313 \\
4 & 2 & 14986 & 4 & 2 & 0.9996 & 0.3333 & 0.5000 & 0.4000 & 0.7068 \\
4 & 2 & 14986 & 4 & 2 & 0.9996 & 0.3333 & 0.5000 & 0.4000 & 0.7068 \\
14 & 5 & 14965 & 15 & 9 & 0.9984 & 0.2500 & 0.3571 & 0.2941 & 0.6841 \\
4 & 4 & 14983 & 7 & 0 & 0.9995 & 0.3636 & 1.0000 & 0.5333 & 0.6809 \\
6 & 3 & 14988 & 0 & 3 & 0.9998 & 1.0000 & 0.5000 & 0.6667 & 0.7313 \\
6 & 3 & 14988 & 0 & 3 & 0.9998 & 1.0000 & 0.5000 & 0.6667 & 0.7313 \\
4 & 3 & 14990 & 0 & 1 & 0.9999 & 1.0000 & 0.7500 & 0.8571 & 0.7313 \\
\hline 6 & 3 & 14982 & 5 & 3 & 0.9994 & 0.6145 & 0.5546 & 0.5320 & 0.7098 \\
$(3.7052)$ & $(1.0541)$ & $(9.1260)$ & $(5.8310)$ & $(2.8717)$ & $(0.0006)$ & $(0.3466)$ & $(0.1884)$ & $(0.1838)$ & $(0.0212)$ \\
\hline
\end{tabular}




\section{DISCUSSION}

This section will discuss and analyze the results presented in Section 6.

\subsection{Experiment 1 and Experiment 2}

In this section, we will plot experiment 1's and experiment 2's results on top of each other. Experiment 1's values, unlabeled data, will be plotted in green. Experiment 2's values, labeled data, will be plotted in red.

\subsubsection{Max}

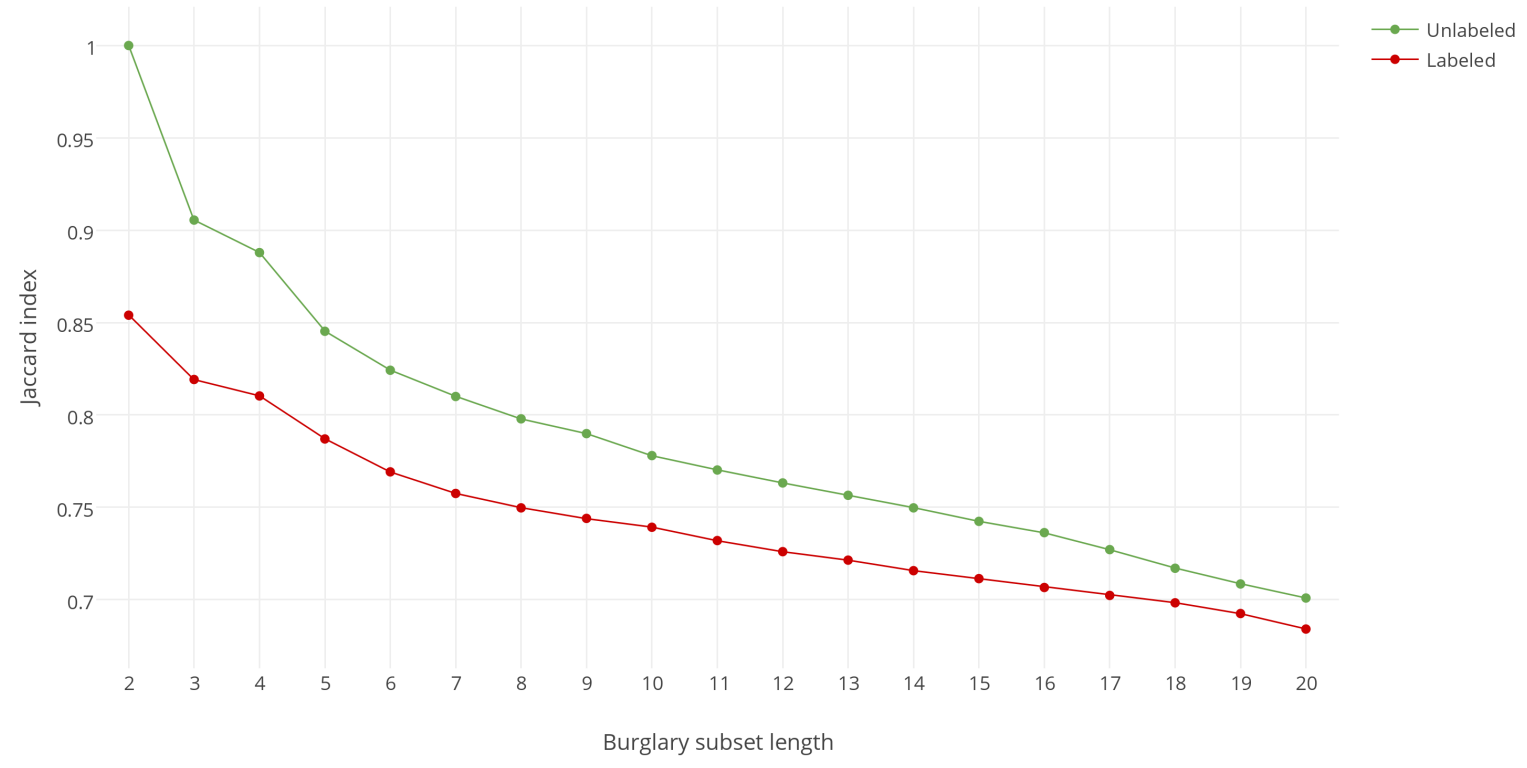

Figure 7.1: Max

Figure 7.1 shows the Max for both experiment 1 and experiment 2. Experiment 1, with unlabeled data, finds more Max values than experiment 2, with labeled data. This could be because experiment 1 has more data to work with than experiment 2 . This shows potential for the algorithm to work unconditionally on unlabeled data trying to find similar crime routes.

The relatively high values for Max indicates that there is burglary subset that might been committed by the same offender, but has not yet been solved. Those burglary subsets with relatively high similarity can be specially interesting for the law enforcement to investigate further.

\subsubsection{Avg top 5}

Figure 7.2 shows the Avg top 5 for both experiment 1 and experiment 2. What is interesting to note is the Avg top 5 results for experiment 2 is slightly higher than the Avg top 5 experiment 1. That could be because of the labeled data used for experiment 2, which probably included more series with more burglaries from the same offender, than experiment 1 . 


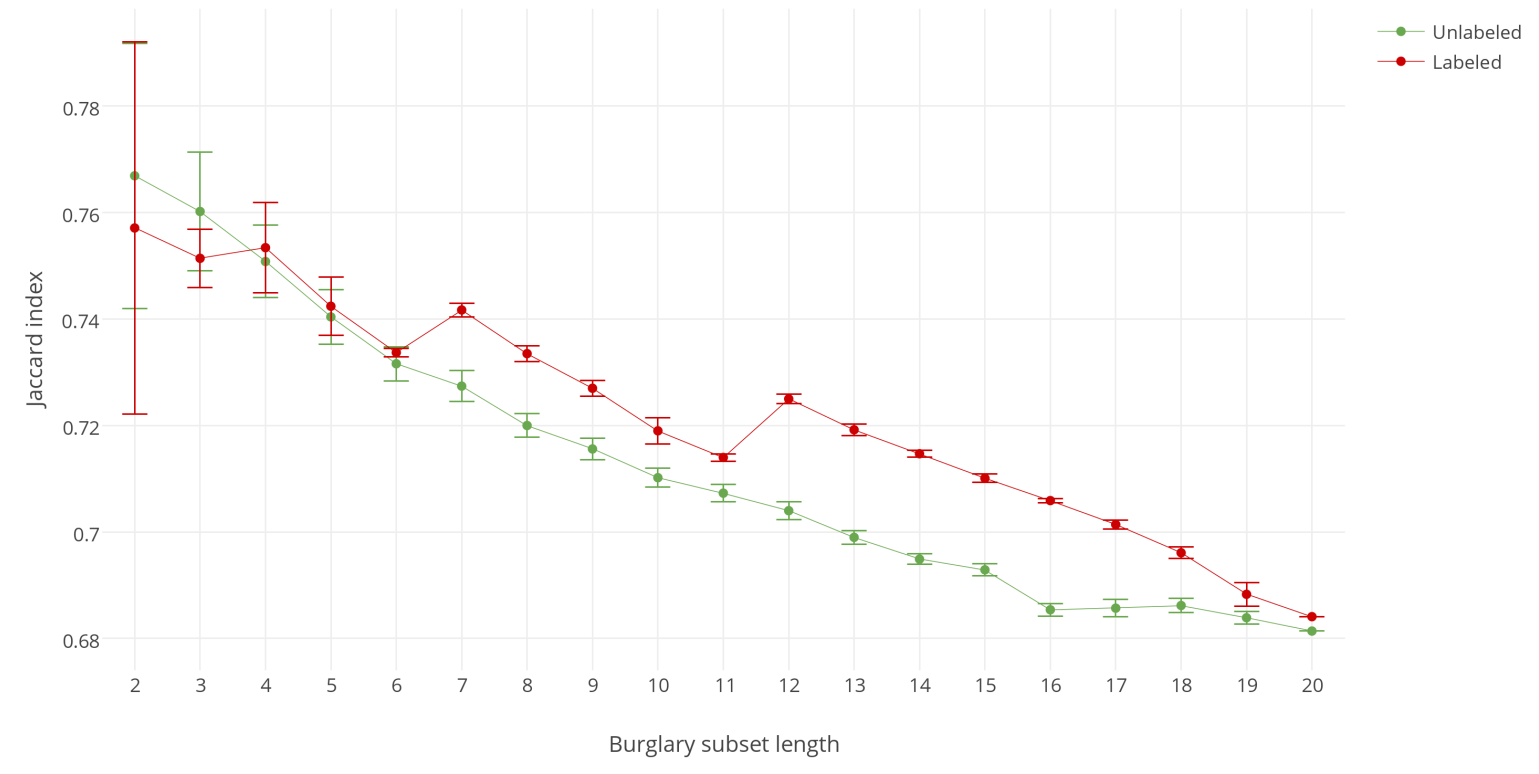

Figure 7.2: Avg top 5

However, burglary subset length of 2 and 3 has a higher Avg top 5 in experiment 1 with the unlabeled data. Since the clearance rate for burglaries in Sweden is below 5\% yearly, it can be that our algorithm is picking up small unsolved burglary series with the same offender. Once the burglary subset length gets higher than 3, experiment 2 has a higher Avg top 5 similarity. The reason to why the algorithm finds higher Avg top 5 for experiment 2 when the burglary subset length is higher than 3 could be because of how we select the timespan for experiment 1 and experiment 2. Experiment 1 uses only one week as a time frame whereas experiment 2 uses the first and last known burglaries as start and end for the timespan, which often is longer than one week. It could be that a serial offender don't do more than 2-3 burglaries under one week.

When the subset length of burglaries gets over 6 the difference in Avg top 5 gets significantly higher for experiment 2. Again, further strengthens the theory that crime series with the same offender has a higher similarity than a random crime route.

\subsubsection{Average}

Figure 7.3 shows the Average for both experiment 1 and experiment 2. Again, experiment 2 has, on average, a higher similarity among crime routes. This further strengthens the theory that serial offender has a higher higher similarity than a random crime route. 


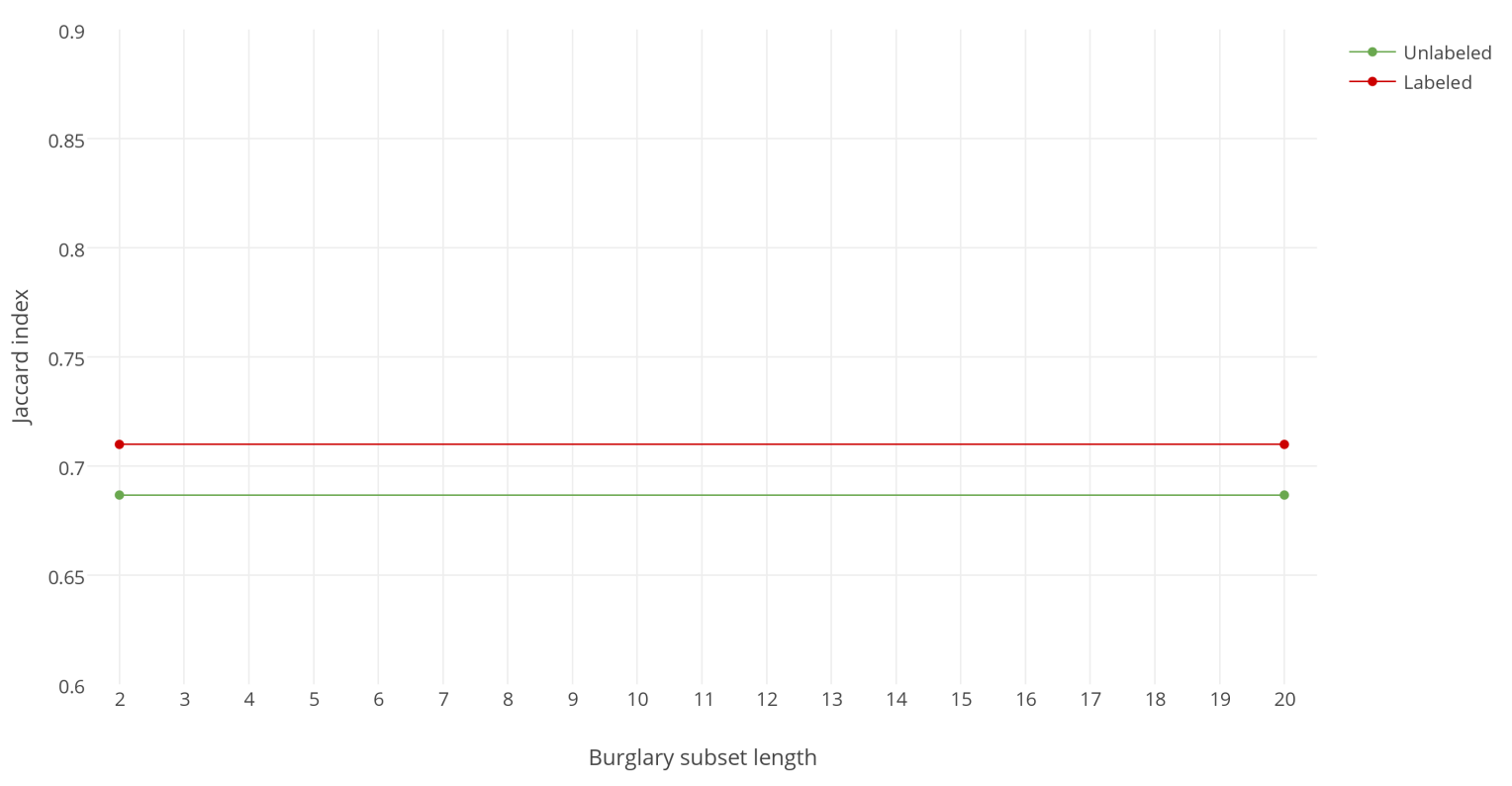

Figure 7.3: Average

\subsection{Experiment 3}

Table 6.3 shows the results for experiment 3. The similarity and the $F_{1}$-score has a Spearman's rank-order correlation value of 0.790 which incidates that the two values are related to each other. This indicates that when the $F_{1}$-score goes up, the similarity also goes up. This is a key finding of this thesis and further strengthens the theory that burglaries with the same offender can be linked using MO features.

To provide further basis for the theory, more similar experiments needs to be conducted with more labeled data. Our experiment had only access to 9 crime series where the offender was known, which could be problematic to draw any significant conclusions from.

The reason to why the algorithm not always performs very well could be because of a number of reasons. It could be because the offender has been active during different time spans on different locations. Other possibilities for improving the algorithm is discussed in section 9 . 



\section{CONCLUSIONS}

Our algorithm is able to find a subset of burglaries with a similarity between the burglaries distinct from a random groups of burglary samples. It has also been shown that the similarity between groups of burglaries are often higher when the same offender figures in two or more burglaries within the group. This is interesting since it strengthens the theory that burglaries with the same offender has a higher similarity than average.

This is interesting for the police in their work of analyzing burglaries. Instead of manually search and analyze combinations of burglaries to find interesting burglaries to focus resources on, they instead can let an application do the initial work for them. By having an application search, analyze and present interesting burglaries the police can make a decision on whether to investigate the burglaries further, or not. Having an application automatically do the initial work of searching and analyzing burglaries will decrease the time and decrease the bias for searching and analyzing burglaries. This enables the police to do more analyzing, through an application, which could lead to better focus on what burglaries to put police resources on. 

The algorithm could be parallelized to decrease execution times. Once all combinations of subsets of burglaries are found, each thread could start analyzing burglary subsets on its own.

In the process of including burglaries in a burglary set, a threshold could be used decide whether a burglary should be excluded or not. If a burglary is below a certain threshold, in terms of similarity, the burglary can be removed. This could improve the FP rates within a burglary set.

When calculating the similarity between burglaries in burglary set, the algorithm first handles missing data. The algorithm does so by excluding MO features that are not present in all burglaries. Further investigation to the amount of MO features that is needed for the similarity result to be representative is needed.

The algorithm in this thesis uses only one route to search for burglaries. The route is decided from the waypoints that come as a input argument to the algorithm. However, the can exist alternative routes through the waypoints. Having the algorithm do analysis on alternative waypoints could be interesting for future work.

The radius value that was used in this thesis, $2 \mathrm{~km}$, is a fixed value. As a future work, it could be interesting to look at other radius values. Both statically values or more dynamic values based on area parameters such as population density.

In this thesis, we have not looked at the distribution of crimes over routes and weeks. To see if certain type of routes performs better than other, or if certain type of time spans performs better than other, is also a topic for future work.

It could also be interesting to replicate this study with more labeled data. Only 9 instances of labeled data fitted out criteria for our experiment. By replicating this study with more labeled data, it could confirm the current results, or shed light on new interesting findings.

At last, it could also be interesting to test the theory that it is possible to find serial offender by looking at MO features on other types for crimes. We have looked at burglaries in this study, but other crimes such a car theft or other types of theft could be interesting to look at. 



\section{REFERENCES}

[1] Residential burglary - brå. http://www.bra.se/bra/bra-in-english/home/ crime-and-statistics/residential-burglary.html. Accessed: 2016-06-06.

[2] Raymond B. and Fosdick. The modus operandi system in the detection of criminals. 5(6): 887-891, 1931.

[3] C Bennell and D V Canter. Linking commercial burglaries by modus operandi: tests using regression and ROC analysis. Science \& justice : journal of the Forensic Science Society, 42(3):153-164, 2002.

[4] Craig Bennell and Natalie J Jones. Between a ROC and a Hard Place: A Method for Linking Serial Burglaries by Modus Operandi. 2005.

[5] W. Bernasco. Them Again?: Same-Offender Involvement in Repeat and Near Repeat Burglaries. European Journal of Criminology, 5(4):411-431, 2008.

[6] Anton Borg, Martin Boldt, Niklas Lavesson, Ulf Melander, and Veselka Boeva. Detecting serial residential burglaries using clustering. Expert Systems with Applications, 41(11): 5252-5266, 2014.

[7] Peter Flach. Machine learning: the art and science of algorithms that make sense of data. Cambridge University Press, 2012.

[8] Natalia Iwanski, Richard Frank, Vahid Dabbaghian, Andrew Reid, and Patricia Brantingham. Analyzing an offender's journey to crime: A Criminal Movement Model (CriMM). Proceedings - 2011 European Intelligence and Security Informatics Conference, EISIC 2011, pages 70-77, 2011.

[9] Paul Jaccard. Distribution de la Flore Alpine: dans le Bassin des dranses et dans quelques régions voisines. Rouge, 1901.

[10] Lucy Markson, Jessica Woodhams, and John W. Bond. Linking serial residential Burglary: Comparing the utility of Modus operandi behaviours, geographical proximity, and temporal proximity. Journal of Investigative Psychology and Offender Profiling, 2010.

[11] Giles Oatley, Brian Ewart, and John Zeleznikow. Decision support systems for police: Lessons from the application of data mining techniques to "soft" forensic evidence. Artificial Intelligence and Law, 2006.

[12] Giles Oatley, Brian Ewart, and John Zeleznikow. Decision support systems for police: Lessons from the application of data mining techniques to "soft" forensic evidence. Artificial Intelligence and Law, 14(1-2):35-100, 2006.

[13] House of Commons. Forensic science on trail, seventh report of session. 2005.

[14] Daniel J. Power. Understanding Data-Driven Decision Support Systems. Information Systems Management, 25(2):149-154, 2008. URL http://www.tandfonline.com/ doi/abs/10.1080/10580530801941124.

[15] J. P. Shim, Merrill Warkentin, James F. Courtney, Daniel J. Power, Ramesh Sharda, and Christer Carlsson. Past, present, and future of decision support technology. Decision Support Systems, 33(2):111-126, 2002.

[16] Herbert A Simon. The new science of management decision. 1960.

[17] Matthew Tonkin, Jessica Woodhams, Ray Bull, John W. Bond, and Emma J. Palmer. Linking different types of crime using geographical and temporal proximity. Criminal Justice and Behavior, 38(11):1069-1088, 2011. 
[18] Jessica Woodhams, Clive R Hollin, and Ray Bull. The psychology of linking crimes: A review of the evidence. 2007. 



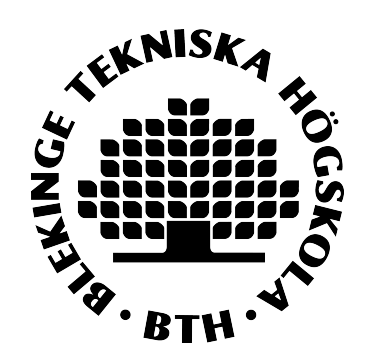

Department of Computer Science and Engineering, Blekinge Institute of Technology, Campus Gräsvik, 37 I 79 Karlskrona, Sweden 